\title{
Shiatsu
}

\section{Die Haut als „Touchpad“}

\author{
Shiatsu ist eine japanische Form der ganzheitlichen Körperbehandlung. \\ Dabei werden direkt unter der Haut verlaufende Energiekanäle per \\ Druckmassage stimuliert, um die körpereigenen Selbstheilungskräfte \\ anzuregen.
}

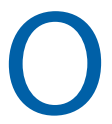
bwohl der Begriff erst zu Beginn des 20. Jahrhunderts geprägt wurde, gehen die Ursprünge auf das China um 530 v. Chr. zurück. Dort hatte der Mönch Bodhidharma ein System zur Gesunderhaltung durch Entgiftung mittels Körperübungen und Selbstmassage entwickelt. Diese Praxis hat sich unter der Bezeichnung Do-In bis in heutige Zeit erhalten. Die als Shiatsu bekannte Technik wird allerdings von einem Behandelnden oder Therapeuten an einer anderen Person ausgeführt.

Das japanische Gesundheits- und Wohlfahrtsministerium definiert Shiatsu als „eine Form der manuellen Behandlung, bei der Daumen, Finger und Handteller, jedoch keine mechanischen oder anderweitigen Instrumente benutzt werden, um Druck auf die menschliche Haut auszuüben. Ziel dieser Behandlung ist es, innere Fehlfunk-

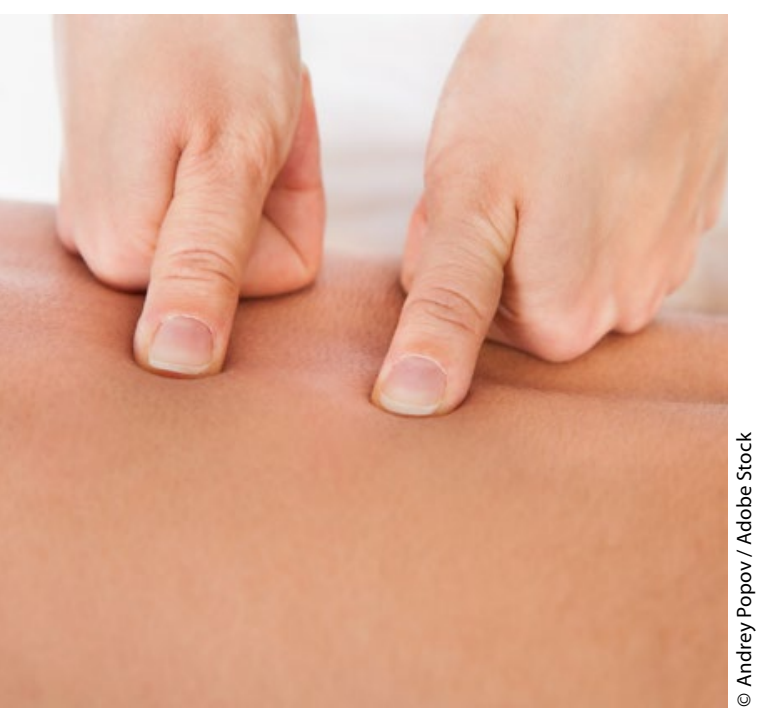

tionen zu korrigieren, um die Gesundheit $\mathrm{zu}$ fördern und zu erhalten und um bestimmte Krankheiten zu behandeln."

In seiner heutigen Form verbindet Shiatsu unterschiedliche Einflüsse: Die ganzheitliche Grundhaltung, basierend etwa auf der Lehre vom „Qi“", der universellen Lebenskraft, der Theorie von Yin und Yang oder die Vorstellung vom menschlichen Meridiansystem ist in Asien verwurzelt. Doch auch das westliche Anatomie- und Körperverständnis, aus dem Techniken wie Dehnungen und Gelenksrotationen hervorgehen, wurde integriert.

\section{Energiegeflecht}

Zugrunde liegt die Vorstellung, dass ein Geflecht von Meridianen den Körper durchzieht. Diese Meridiane sind nicht deckungsgleich mit den Blutbahnen vielmehr leiten sie die Energie, das Qi, durch den Körper und verbinden die unterschiedlichen Körperfunktionen miteinander. So verläuft etwa der Dickdarmmeridian, sehr vereinfacht dargestellt, von den Nasenflügeln über die Schultern bis zu den Spitzen der Zeigefinger. Der Blasenmeridian wiederum beginnt bei der Nasenwurzel und verläuft über Hinterkopf, Rücken und die Rückseite des Beines hinab bis zur Außenseite des kleinen Zehs.

Ist die Zirkulation des Qi im Körper gestört, können Krankheiten auftreten. Durch sanften Druck von außen werden die Meridiane stimuliert, um Blockaden zu lösen, Selbstheilungskräfte des Körpers zu stärken und ganz allgemein das körperliche und seelische Befinden zu unterstützen. Shiatsu wird deshalb ins- besondere auch als Präventivbehandlung eingesetzt.

Shiatsu bedeutet übersetzt „Fingerdruck“. Tatsächlich aber setzt der Behandelnde nicht nur die Finger ein, sondern auch Handballen, Knöchel, Ellbogen, Knie oder Füße. Zudem können Meridiane durch gezieltes Drehen und Dehnen bestimmter Körperpartien stimuliert werden.

Die Haut wird somit zum Touchpad zur Membran, die Druck, heilende Informationen an darunterliegende Schichten vermitteln soll. In der Tiefe des Körpers, auf der „Festplatte“, findet dann die Veränderung statt: Über die Haut wird somit der Energiefluss im Körper angeregt beziehungsweise ausgeglichen.

\section{Behandlung durch Körper und Geist}

Anders als bei Formen der reinen Entspannungsmassage oder der „klassischen" Sportmassage zur Auflockerung von Muskelpartien handelt es sich beim Shiatsu um eine energetische Körperarbeit mit ganzheitlichem Ansatz. Ein ausführliches Erstgespräch und genaue Befundung bilden die Grundlage der Behandlung - berücksichtigt werden alle Faktoren, die für die Konstitution des Patienten relevant sind: von (akuten) körperlichen Beschwerden über seelische Belastungen bis hin zu einschneidenden Veränderungen in Beruf oder Familienleben.

Haltung und Fokussierung der Therapeuten spielen eine entscheidende Rolle. Die Behandlung erfolgt weniger durch Muskelkraft als mittels Körpergewicht und energetischer Konzentration auf den Patienten: Weniger als eine „technische" Ausführung steht die achtsame Berührung im Vordergrund.

Durch tiefe Entspannung wird die Atmung freier und das Immunsystem kräftiger, Selbstwahrnehmung und das vertrauensvolle Sich-Überlassen werden geschult.

Myrta Köhler 